Man and Nature

L'homme et la nature

\title{
Les femmes et la diffusion des Lumières
}

\section{Béatrice Didier}

Volume 7, 1988

URI : https://id.erudit.org/iderudit/1011925ar

DOI : https://doi.org/10.7202/1011925ar

Aller au sommaire du numéro

Éditeur(s)

Canadian Society for Eighteenth-Century Studies / Société canadienne d'étude du dix-huitième siècle

ISSN

0824-3298 (imprimé)

1927-8810 (numérique)

Découvrir la revue

Citer cet article

Didier, B. (1988). Les femmes et la diffusion des Lumières. Man and Nature / L'homme et la nature, 7, 23-52. https://doi.org/10.7202/1011925ar

Copyright (c) Canadian Society for Eighteenth-Century Studies / Sociéte canadienne d'étude du dix-huitième siècle, 1988
Ce document est protégé par la loi sur le droit d'auteur. L'utilisation des services d'Érudit (y compris la reproduction) est assujettie à sa politique d'utilisation que vous pouvez consulter en ligne.

https://apropos.erudit.org/fr/usagers/politique-dutilisation/ 


\section{Les femmes et la diffusion des Lumières}

Parler des femmes et de la diffusion des Lumières. Beau sujet, certes, mais qui risque d'être à la fois un peu rebattu et trop vaste. Or mes inquiétudes, sur le premier point du moins, ont assez vite été dissipées. $\mathrm{Au}$ fond, si étrange que cela puisse paraître, le sujet n'avait ni été dégrossi dans ses grandes lignes, ni vraiment traité en détail. Bien entendu, on a beaucoup écrit sur les femmes au XVIIIe siècle, mais presque toujours, jusqu'à ces toutes dernières années, ce sont des hommes qui ont fait ces études, et avec la mentalité de leur temps. C'est-à-dire que ce qui intéressait, ce qui semblait même assez croustillant, c'était l'histoire des diverses liaisons de telle ou telle avec un grand écrivain. De même pendant longtemps on ne s'était intéressé à George Sand que parce qu'elle avait couché avec Chopin ou Musset! Que s'était-il passé entre Mlle de Lespinasse et d'Alembert, entre Mme du Châtelet et Voltaire: voilà à quoi se limitait l'intérêt de beaucoup de critiques. L'aspect mondain de la question les attirait aussi: ces dames avaient des salons: quel était leur 'jour'? qui y venait? etc., ce qui est déjà plus intéressant, mais demeure un peu superficiel. L'étude des Goncourt, si passionnante, si neuve soit-elle, ne parvient pas à sortir absolument de cet état d'esprit qui est celui de la fin du XIXe siècle, et qui d'ailleurs se prolonge largement dans la première moitié du XXe siècle, où, si telle ou telle étude apporte beaucoup sur une femme en particulier, l'ensemble de la réflexion sur les femmes au XVIIIe siècle, s'enferme toujours dans l'univers étroit des salons et des liaisons, à l'exception du livre fort intéressant de L. Abensour. ${ }^{1}$ Deux thèses dans ces dernières années ont apporté du neuf: celle de P. Hoffmann, $\mathrm{La}$ femme dans la pensée des Lumières (Ophrys, 1977), et celle de P. Fauchery, La destinée féminine dans le roman européen du XVIIIe siècle (Colin, 1972): deux très beaux sujets certes, mais qui ne recouvrent pas exactement le champ que nous nous proposons de survoler aujourd'hui. Le récent livre d'E. Badinter plein d'aperçus stimulants (Émilie, Émilie: l'ambition féminine au XVIIIe siècle [Flammarion, 1983]) n'aborde que de biais la question des femmes et de la diffusion des Lumières, quoique pour cette question la personnalité de Mme du Châtelet soit déterminante. 
Notons enfin que nous avons des intruments de travail qui manquaient aux études de la fin du XIXe ou de la première moitié du XXe siècle. Des éditions scientifiques des textes de femmes, et surtout des publications sérieuses de leurs correspondances sont évidemment indispensables; elles se constituent petit à petit. ${ }^{2}$ Et la connaissance que nous pouvons avoir des femmes du XVIIIle siècle a bénéficié très largement des progrès qu'ont accompli les études sur le siècle des Lumières.

Le sujet de notre conférence n'en demeure pas moins redoutable, car son étendue provient non seulement de la masse des matériaux, mais, ce qui est plus ennuyeux, d'un point de vue méthodologique, de la difficulté de définir les termes de départ. Cela est si évident que je me contenterai d'un rapide rappel. Qu'est-ce que les Lumières? Des équipes de dix-huitiémistes se sont efforcées de répondre à cette question. Un numéro de la revue XVIIIe siècle a posé les données du problème, ${ }^{3}$ sans prétendre évidemment le résoudre. La simple question des dates fait problème. Ce n'est pas au même moment que combattent et triomphent les Lumières dans tous les pays d'Europe. Nous nous en tiendrons ici à la France, tout en reconnaissant que, dans un mouvement d'idées si largement cosmopolite, il est assez arbitraire de se laisser arrêter aux frontières. Mais nous n'avons qu'une heure devant nous! En France même, quelles dates choisir: 1715-1789 sont des dates simples, parce que reposant sur des événements historiques incontestables, mais évidemment elles ne rendent pas compte de la souplesse de la vie littéraire et intellectuelle. En tout cas, il serait impossible de se limiter aux années 1750-60 comme étant le moment du démarrage de l'Encyclopédie. L'appartenance à l'Encyclopédie, n'est pas un critère suffisant, à la fois parce que l'attribution des articles pose d'énormes problèmes, et parce que des figures très marquantes des Lumières n'ont que fort peu ou pas du tout participé à l'entreprise (Montesquieu, Voltaire). Pour notre sujet, en particulier, si les femmes ont été fort liées aux Encyclopédistes, il ne semble pas qu'elles aient rédigé des articles. Mais elles ont pu contribuer à fournir une documentation sur certains sujets (tissus, modes, médecine). On est obligé de s'en tenir pour définir les Lumières à des critères plus généraux, plus idéologiques, et par conséquent plus vagues. Finalement, c'est encore la classique définition de Kant qui serait la plus opérante:

Les Lumières sont ce qui fait sortir l'homme de sa minorité qu'il doit s'imputer à lui-même. La minorité consiste dans l'incapacité où il est de se servir de son intelligence sans être dirigé par autrui. Il doit s'imputer à lui-même cette minorité quand elle n'a pas pour cause le manque d'intelligence, mais l'absence de résolution et du courage nécessaire pour user de son esprit sans être guidé par un autre. Sapere aude, aie le courage de te servir de ta propre intelligence! Voilà donc la devise des Lumières. (Was ist aufklarung?) 
Cette définition des Lumières, si éclairante par elle-même, nous amène d'emblée au vif de notre sujet. La femme est considérée comme mineure jusqu'à une époque récente. Sa situation juridique au XVIIIe siècle est bien celle d'une mineure, et nous allons voir comment le courant philosophique des Lumières pour elles, plus encore que pour l'homme - et en quelque sorte à un double titre - consiste en une accession vers l'âge adulte, accession lente d'ailleurs, et que le XVIIIe siècle ne pourra réaliser complètement: l'élan cependant aura été donné. Il semblerait que le mot 'femmes' soit plus facile à définir que celui de 'Lumières,' si des années de recherche féministe ne nous avaient instruit sur les pièges innombrables que comporte toute tentative de définition. S'en tient-on à un critère physiologique? Mais alors on néglige que la féminité est aussi un phénomène culturel, et combien prégnant dans une société d'Ancien Régime où le clivage homme/femme est si déterminant dans la répartition des rôles, à tous les niveaux de la société. Se cantonne-t-on dans l'étude du phénomène culturel, on risque de négliger la spécificité physiologique si importante, surtout à une époque où le sensualisme, les progrès de la médecine amènent une redécouverte du corps tandis que le déterminisme physiologique pèse encore si lourdement sur la femme. On ne sort pas facilement du dilemme Nature/Culture, dilemme qui justement a été fortement marqué par la réflexion philosophique des Lumières.

À la fois 'naturel' et culturel, le clivage homme/femme est, bien évidemment, une des rares constantes qui traverse tous les degrés de la société si étroitement hiérarchisée sous l'Ancien Régime, le clivage commun à tous les 'Ordres,' Noblesse, Tiers État, et Clergé. Y a-t-il pour autant une cause commune des femmes? La question de l'allaitement rapproche-t-il la paysanne de l'aristocrate, du moins à partir du moment où celle-ci suit la mode de nourrir son enfant? À vrai dire, nous abordons là un secteur très mal connu, celui des classes populaires au XVIIIe siècle. Dans quelle mesure les Lumières ont-elles pénétré dans les couches les plus défavorisées de la population, dans quelle mesure les femmes de ces classes, qui avaient tout intérêt à ce progrès, y ont-elles participé? Il y aurait là de quoi faire des études passionnantes qui relèvent d'ailleurs plus de l'histoire des faits sociaux que de l'histoire de la littérature. La femme du peuple n'écrit pas - l'homme non plus. Encore faudrait-il explorer systématiquement les livres de raison, les cahiers de doléances (rares) de corporations féminines à Paris. Nous devons reconnaître que nous nous servons ici surtout de lettres, de textes littéraires rédigés par des femmes qui appartiennent à l'aristocratie ou à la grande bourgeoisie. Mais nous voulions cependant évoquer cette question de la diffusion possible d'aspirations qui 
se rattachent à l'idéologie des Lumières, dans les classes sociales défavorisées et du rôle que les femmes de ces classes peuvent avoir eu.

On s'en doute, en voyant l'horloge tourner, nous ne pouvons ici que poser les jalons d'une problématique, énumérer les questions qu'il serait utile d'aborder, peut-être en susciter d'autres. Quant à traiter à fond ce passionnant sujet: 'Les femmes et la diffusion des Lumières,' il y faudrait plusieurs thèses, et des recherches qui émaneraient de chercheurs appartenant à des spécialités fort diverses. Nous rappellerons d'abord la place de la femme dans le système que construisent les Philosophes, nous verrons ensuite le rôle des femmes dans la mêlée: en quoi ont-elles participé au combat, pour nous demander finalement s'il y a des Lumières au féminin si l'on peut dire; quelle spécificité la présence des femmes a-t-elle apporté à ce mouvement sollicité par l'universalisme?

I

Le discours des Encyclopédistes sur les femmes n'est évidemment pas absolument neuf et on y voit des reliquats de mentalité ancienne, avec cependant des perspectives nouvelles. L'article 'Femme (anthropologie)' de l'Encyclopédie est intéressant par un mélange d'audaces et de prudence. Sur la question de la différence sexuelle, il pose la question un peu piégée d'avance de savoir qui des deux sexes est premier. Mais, loin de considérer la femme comme ayant un facteur négatif, un moins, il reprend l'opinion de Daubenton selon laquelle au contraire la matrice est en plus, 'la matrice qui est de plus dans les femmes que dans les hommes.' Il s'indigne de la carence de l'éducation: 'On a si fort négligé l'éducation des femmes chez tous les peuples policés, qu'il est surprenant qu'on en compte un aussi grand nombre $d$ 'instruites par leur érudition et leurs ouvrages.' L'article est signé 'G.' Ce qui frappe, c'est la diversité du discours sur la femme d'un article à un autre, diversité qui prouve la complexité du sujet et un certain désarroi des Lumières devant le 'continent noir.' L'article 'Femme (morale)' a un son de cloche assez traditionnel, et tombe dans les écueils que présente toujours le thème du 'mystère' féminin: 'Les femmes ne diffèrent pas moins des hommes par le coeur et par l'esprit, que par la taille et par la figure; mais l'éducation a modifié leurs dispositions naturelles en tant de manières, la dissimulation qui semble être pour elles un devoir d'état, a rendu leur âme si secrète, les exceptions sont en si grand nombre, si confondues avec les généralités, que plus on fait d'observations, moins on trouve de résultats.' Encore ce texte a-t-il le mérite d'amorcer des réflexions critiques sur l'éducation des couvents et la 
société qui sont développées de façon plus explicite par la suite: 'Des femmes qui ont renoncé au monde avant que de le connaître, sont chargées de donner des principes à celles qui doivent y vivre. C'est de là que souvent une fille est menée devant un autel, pour s'imposer par serment des devoirs qu'elle ne connaît point, et s'unir pour toujours à un homme qu'elle n'a jamais vu.'

L'article le plus audacieux est peut-être celui de Jaucourt 'Femme (droit nat.),' parce qu'il pose la question non plus en termes de physiologie ou de psychologie, mais dans ses implications politiques. Certes il part de l'enseignement traditionnel de l'Église se contentant d'utiliser le terme d'Être suprême, pour éviter une référence trop nette au catholicisme, mais c'est finalement pour aévelopper l'idée que la fin du mariage est la procréation, après avoir quelque peu paraphrasé la Genèse:

L'Être suprême ayant jugé qu'il n'était pas bon que l'homme fût seul, lui a inspiré le désir de se joindre en société très étroite avec une compagne, et cette société se forme par un accord volontaire entre les parties. Comme cette société a pour but principal la procréation et la conservation des enfants qui naîtront, elle exige que le père et la mère consacrent tous leurs soins à nourrir et à bien élever ces gages de leur amour, jusqu'à ce qu'ils soient en état de s'entretenir et de se conduire eux-mêmes.

Derrière ce discours traditionnel apparaissent des audaces. Il est suggéré par la phrase même que nous venons de citer que le mariage n'a pas absolument à être indissoluble, que cette indissolubilité peut se réduire à la période de l'éducation des enfants. Mais surtout ce texte, comme l'ensemble de l'article de Jaucourt, introduit la notion de contrat, si importante dans la pensée politique. Certes, elle n'est pas étrangère à la théologie chrétienne pour qui la validité du mariage repose sur l'accord des parties. Mais elle change ici d'optique. De même qu'il y avait déjà eu des théoriciens du contrat parmi les défenseurs de l'absolutisme, et que l'audace d'un Rousseau ou d'un Diderot, consiste à aller jusqu'au bout et à prétendre que si les conditions du contrat ne sont pas remplies, le peuple est libre de reprendre le pouvoir, ici l'audace va consister à remettre en cause l'habitude de donner le pouvoir au mari. On entendu dans ce texte de façon contradictoire deux voix du despotisme éclairé, au masculin et au féminin. En ce sens qu'au début est rappelé le code de Frédéric II paru en 1750, mais que plus loin l'exemple de Catherine II va prouver que les femmes peuvent fort bien exercer le pouvoir. Le pouvoir masculin n'est pas de nature, mais de convention. 
Il serait difficile de démontrer que l'autorité du mari vienne de la nature; parce que ce principe est contraire à l'égalité naturelle des hommes ... On peut donc soutenir qu'il n'y a point d'autre subordination dans la société conjugale, que celle de la loi civile, et par conséquent rien n'empêche que des conventions particulières ne puissent changer la loi civile, dès que la loi naturelle et la religion ne déterminent rien au contraire.

La suite de l'article de Jaucourt va démontrer par des exemples historiques que les femmes peuvent très bien régner sur un empire ou sur leur ménage:

L'exemple de l'Angleterre et de la Moscovie fait bien voir que les femmes peuvent réussir également et dans le gouvernement modéré et dans le gouvernement despotique; et s'il n'est pas contre la raison et contre la nature qu'elles régissent un empire, il semble qu'il n'est pas contradictoire qu'elles soient maîtresses dans une famille.

Viennent à l'appui l'exemple de Lacédémone et des Égyptiens. Pour revenir enfin sur cette notion centrale de contrat:

Le mariage est de sa nature un contrat; et par conséquent dans tout ce qui n'est point défendu par la loi naturelle, les engagements contractés entre le mari et la femme en déterminent les droits réciproques.

L'article 'Femme en couche' est également de Jaucourt et il contient des conseils fort judicieux, compte tenu des connaissances médicales de l'époque. Ce qui montre bien aussi le progrès des Lumières, c'est ce désir de médicaliser l'accouchement. Les maladies des femmes en couche relèvent du médecin:

ce traitement appartient de droit aux Médecins; les Accoucheurs n'y doivent paraître qu'en sous-ordre, et toujours proportionnellement à l'étendue de leurs lumières en Médecine; si elles sont supérieures en ce genre, tout parle en leur faveur, tout conspire à leur rendre hommage dans cette conjoncture.

On lit dans ce texte la tendance de l'Encyclopédie à rattacher la chirurgie à la Médecine, et à s'écarter des habitudes archaïques où la chirurgie était parfois exercée par un simple barbier.

Quand on voit l'audace, la nouveauté des articles de Jaucourt dans le domaine juridique et même dans le domaine médical, on n'en est que plus étonné de voir malgré tout la survivance de discours archaïque dans d'autres articles. Prudemment le chevalier de Jaucourt refuse de s'attarder à la question de la 'passion hystérique' et renvoie 
à un autre article. De même, à l'article 'menstruel,' il élimine tout discours magique: 'On sait que toutes ces fausses idées sont le fruit de l'ignorance, et qu'une femme qui se porte bien ne rend point un sang menstruel différent de celui qui circule dans les artères du reste du corps.' Même saine doctrine dans l'article 'Vapeurs' (non signé) qui refuse de croire que les vapeurs sont des émanations de la matrice et parle également des vapeurs des jeunes gens, en proposant comme remède l'hygiène et le travail: 'les vapeurs attaquent surtout les gens oisifs de corps.'

Mais que pense le maître d'oeuvre? Diderot a eu de nombreuses occasions de s'exprimer sur un sujet aussi vaste. Néanmoins nous retiendrons surtout les deux textes que lui inspire le livre de Thomas Essai sur le caractère, les moeurs et l'esprit des femmes dans les différents siècles. Il s'agit en fait d'un seul texte, mais sous deux versions différentes, la première, moins développée, est une copie manuscrite de la Correspondance littéraire (ler avril 1772), la seconde est celle qui parut dans l'édition Naigeon; par rapport à la première version, elle s'enrichit de beaucoup de réflexions ethnologiques. Il s'agit, comme aime à le faire Diderot, de beaucoup plus que d'un compte rendu du livre de Thomas qu'il accuse de n'être d'aucun sexe; le texte de Diderot, au contraire, a un sexe: il est écrit par un homme que fascine et inquiète le monde de la femme. ${ }^{4}$ La femme est représentée comme étant par excellence l'être des fortes passions. 'C'est surtout dans la passion de l'amour, dans les transports de la jalousie, dans les accès de la tendresse maternelle, dans la superstition, dans la manière dont elles éprouvent les émotions épidémiques et populaires que les femmes étonnent.' La femme par excellence, c'est la Pythie, et ajoute Diderot avec quelque humour: 'Jamais un homme ne s'est assis à Delphes sur le trépied.' L'orgasme féminin est représenté comme un phénomène extraordinaire qui transporte hors du temps:

La femme porte au-dedans d'elle-même un organe susceptible de spasmes terribles, disposant d'elle et suscitant dans son imagination des fantômes de toute espèce. C'est alors qu'elle revient sur le passé, qu'elle s'élance dans l'avenir, que tous les temps lui sont présents. Rien de plus contigu que l'hystérisme, l'extase, la révélation, la poésie odaîque et la prophétie. (32)

Dans cette géographie littéraire qui est en train de s'organiser et qui aboutira à la distinction romantique entre littératures du Nord et littératures du Midi, la femme s'apparenterait davantage au génie du Nord. Très caractéristique, cette double référence: les femmes sont belles comme des séraphins de Klopstock, terribles comme des diables de Milton' (38). Intéressantes aussi les réflexions sur le mysticisme 
des femmes. Tandis que Grimm ajoute une note sarcastique sur la facilité des femmes à diffuser le mensonge: 'l'établissement des religions commence ordinairement par les femmes' (35), Diderot analyse le mysticisme des femmes avec beaucoup plus de profondeur et prouve, par le fait même, qu'il connait mieux les écrits mystiques qu'on ne serait tenté de le croire: 'La Guyon a dans son livre des Torrents, des lignes d'une éloquence dont il n'y a point de modèles. C'est SainteThérèse qui a dit des démons: 'Qu'ils sont malheureux! ils n'aiment point' (42).

La femme appartient au domaine de l'instinct; et à plusiers reprises l'image de la 'bête féroce' revient sous la plume de Diderot:

La femme dominée par l'hystérisme semble éprouver je ne sais quoi d'infernal ou de céleste. Quelquefois elle m'a fait frémir. C'est sous l'action de cette bête féroce qui fait partie d'elle-même, qu'il faut la voir et l'entendre. Comme elle sent! Comme elle s'exprime! Elle ne dit rien qui soit d'une mortelle. (33)

Cette admiration de Diderot pour la primitivité, le sauvage, le 'cri animal de la passion' accroît la fascination que provoquent les femmes. Mais, si belles que soient ces pages, elles sont finalement beaucoup moins audacieuses que celles de Jaucourt dans l'Encyclopédie, car lorsque l'on aborde la question de la femme dans la société, on sent que Diderot n'est pas prêt à remettre en cause le pouvoir masculin. Certes, il reconnaît que le sort de la femme est malheureux, mais, dit-il, il est encore bien pire chez les peuples primitifs et dans la version plus développée de cet article, il ajoute des exemples qui vont dans ce sens. Ainsi tout le triste discours de la femme indienne qui explique au père Jésuite qu'il eût beaucoup mieux valu qu'elle meure à la naissance. Il ajoute aussi le paragraphe sur les îles Mariannes, pour affirmer que la force de l'homme lui assure un suprématie 'naturelle.' 'Partout, excepté aux îles Mariannes, on a trouvé la femme soumise à l'homme.' Le matriarcat est traité d"absurdité' (46).

Certes la vie de la femme, même dans les nations 'policées' est fort triste. Et Diderot trace un tableau des diverses étapes: premières règles, mariage, grossesse, ménopause, qui n'a rien de réjouissant. 'La cruauté des lois civiles s'est réunie contre elles dans presque toutes les contrées à la cruauté de la nature' (34). Les femmes passent d'une 'tyrannie,' celle du père, à une autre tyrannie, celle de l'époux, autrement redoutable puisqu'elle suppose une servitude sexuelle: 'J'ai vu une femme honnête frissonner d'horreur à l'approche de son époux; je l'ai vue se plonger dans le bain, et ne se croire jamais assez lavée de la souillure du devoir' (38). Dans un élan de compassion Diderot s'écrie: 'Femmes, que je vous plains! Il n'y avait qu'un dédommage- 
ment à vos maux, et si j'avais été législateur, vous l'eussiez obtenu. Je vous aurais affranchies, je vous aurais mises au-dessus de la loi; vous auriez été sacrées, en quelque endroit que vous vous fussiez présentées' (34). Mais plutôt que de les mettre au-dessus de la loi, ne vaudrait-il pas mieux prévoir une loi, qui leur assure de véritables droits?

Pour ce qui est du génie féminin dans la création, Diderot n'en parle qu'assez allusivement, dans ce texte du moins. Elles sont les reines de l'intuition: 'il faut convenir que leur ignorance les dispose à recevoir promptement la vérité quand on la leur montre. Elle perce plus difficilement en nous où elle trouve à l'entrée de nos crânes Platon, Aristote, Épicure, Zénon, en sentinelle et armés de piques pour les repousser' (35). Et le dernier mot est certes prometteur - mais le texte s'arrête là: 'Quand elles ont du génie, je crois qu'elles en ont l'empreinte plus originale que nous' (36). En tout cas, le contact des femmes sera utile au génie masculin et Diderot souligne les avantages du commerce des femmes pour un homme de lettres (35). Leur influence sur les créations masculines lui semble incontestable:

Elles nous accoutument encore à jeter de l'agrément et de la clarté dans les matières les plus délicates et les plus épineuses. On leur adresse sans cesse la parole, on veut en être écouté et entendu, on craint de les fatiguer et de les ennuyer; et l'on prend une habitude particulière de s'exprimer qui passe de la conversation dans le style. (35-36)

L'article de Diderot est bien caractéristique - à la fois par ses audaces, mais aussi par une certaine prudence à l'endroit de 'ces enfants bien extraordinaires' (33) dès que l'on aborde le domaine des institutions - de l'attitude des Lumières envers la femme, quoiqu'il soit difficile de parler globalement des 'Lumières,' et qu'il y ait des féministes plus affirmés, par exemple Jaucourt ou surtout la Mettrie.

On voit très bien en quoi la question de la femme intéresse au plus haut point les Lumières. Elle fait partie des opprimés, et comme telle, elle a droit à une libération - assez relative d'ailleurs, comme est assez relative la liberté que les Lumières réclament pour le pauvre ou pour le noir. Elle fascine aussi les Philosophes, comme l'autre de la Raison, comme les fascine la folie, d'une façon qu'a fort bien analysée Michel Foucault:

On laisse revenir en plein jour un personnage de déraison, au moment même où on pensait l'avoir le plus profondément caché dans l'espace de l'internement. Comme si la raison classique admettait de nouveau un voisinage, un rapport, une quasi-ressemblance entre elle et les figures de la déraison. On 
dirait qu'à l'instant de son triomphe, elle suscite et laisse dériver, aux confins de l'ordre un personnage dont elle a façonné le masque à sa dérision - une sorte de double où elle se reconnaît et se révoque à la fois. (Histoire de la folie à l'âge classique)

La femme, comme le fou, l'irrationalité cernant la rationalité, est nécessaire aux Lumières. Mais enfermer la femme dans l'irrationalité n'estce pas encore une manifestation de ce 'grand Refermement' classique?

Il fallait rappeler ces ambiguités de la conception que les hommes des Lumières ont de la femme avant d'en venir à l'analyse de la place que les femmes eurent dans la bataille philosophique. Place qui est ellemême assez difficile à évaluer en profondeur. La question qui a été le plus étudiée, et qui n'est d'ailleurs pas négligeable, c'est le rôle des Salons. Sans le sous-estimer, j'aimerais cependant ne pas réduire mon étude à cet aspect, qui, s'il est important, ne me semble cependant pas couvrir complètement le champ, bien vaste, qui m'a été proposé.

En tout cas, si l'on ne peut guère contourner la question des Salons j'aimerais au moins souligner que l'on a trop souvent limité leur étude à quelques-uns d'entre eux, les plus brillants, ceux de la capitale, tenus par des femmes dont les liens avec les écrivains des Lumières sont évidents. Or dans ce progrès des mentalités, si on l'envisage dans toute son ampleur, des salons beaucoup plus obscurs ont eu un rôle. En particulier les salons de province. On est en train de s'intéresser à la province au temps des Lumières, on a étudié le rôle des Académies; mais ici, comme à Paris, le rôle des Académies est inséparable de celui des Salons. Or ils sont très actifs, ainsi dans des villes comme Lyon, Bordeaux, ou même Autun, Dijon. Les femmes de ces parlementaires, par exemple, qui possèdent de si beaux exemplaires de l'Encyclopédie dans leurs bibliothèques (tout un travail a été fait depuis quelque temps sur les inventaires de bibliothèques privées), ces femmes, donc, tiennent des salons. Nous avons la mauvaise habitude dans notre France traditionnellement centralisée, de parler avec une pointe de mépris de l'érudit local ou de la muse de province, ils ont pourtant beaucoup fait pour la diffusion des Lumières dans la 'France profonde.' Nous ne pouvons citer que quelques noms, mais il y aurait lieu à des études détaillées. Les salons bordelais sont actifs et brillants, ainsi celui de Mme d'Aiguillon qui était la nièce du maréchal de Richelieu. Ou encore, toujours à Bordeaux, le salon de Mme d'Egmont, et surtout, le mieux connu des salons bordelais, celui de Mme Duplessy qui est elle- 
même poète, que fréquente d'autres femmes poètes, ainsi Mme de Pontant-Belhade, mais aussi des personnalités aussi brillantes que Montesquieu et Joseph Vernet. ${ }^{5}$ Toulouse et ses environs sont actifs. La présidente de Bourg tient un salon où se rencontrent poètes et archéologues - il faut se rappeler combien a été important ce travail des archéologues, souvent provinciaux, dans les progrès de l'Histoire au XVIIIe siècle, progrès qui est justement un des aspects de la pensée des Lumières. Dans les salons toulousains on lit Rousseau avec transport, on le commente comme une nouvelle Bible. ${ }^{6} \mathrm{Il}$ faudrait encore citer Lyon, et le salon de Mlle de la Rochebaron où vint Voltaire. Si les Lumières ont certainement beaucoup contribué au rayonnement de Paris en France et dans toute l'Europe, elles sont loin cependant d'être exclusivement un phénomène parisien.

Mais, bien évidemment, quand on parle des salons au XVIIIe siècle on pense à ceux de Mme de Lambert, de Mme de Tencin, de Mme du Deffand, de Mme d'Epinay, de Mme Geoffrin, de Mlle de Lespinasse, de Mme Helvétius, de Mme Necker. Eux, en revanche, ont été beaucoup étudiés. Mais il est bien simplificateur de vouloir les englober dans le même mouvement. Chaque salon possède sa physionomie, même si les invités se retrouvent souvent d'un salon à un autre, physionomie qui tient certes à la personnalité de la maîtresse de maison, et au choix qu'elle opère dans ses invités, mais aussi à ses origines sociales qui sont d'une grande diversité. Mme de Lambert, Mme d'Aiguillon, Mme de Robecq, Mme du Deffand appartiennent à l'aristocratie, à la partie de l'aristocratie qui se considère comme supérieure: la noblesse d'épée; leurs familles ont une place importance à la Cour; elles sont tout près des rouages du pouvoir. Les financiers, les fermiers généraux considèrent qu'avoir un salon grâce à leur femme, augmente ce que nous appelons le 'standing.' Quelque soit la faiblesse ou l'inexistence du lien conjugal, chez plusieurs d'entr'elles, ce n'en est pas moins la finance qui subvient, somptueusement, aux frais qu'entraîne un salon, pour Mme d'Helvétius, Mme d'Epinay, Mme de la Poplinière, Mme Dupin. Mme Geoffrin est une simple bourgeoise.

Diverses dans leur origine, les femmes le sont aussi dans leurs opinions, et l'attitude de ces femmes à l'endroit des Lumières est loin d'être uniforme. Mme de Tencin et Mme de Lambert hésitent à se lancer dans le combat philosophique: la littérature pure les intéresse davantage que la politique. ${ }^{7}$ Cependant le salon de Mme de Lambert que fréquente Marivaux et qu'il évoque dans la Vie de Marianne, est gagné aux Modernes, dans la fameuse querelle qui vite déborde la seule littérature. Elles sont néanmoins assez réservées face aux idées nouvelles. Il y a plus: une Mme du Deffand ne cache pas qu'elle est franchement hostile aux doctrines philosophiques. ${ }^{8}$ Elle n'en contribue pas moins 
cependant à la diffusion des Lumières, puisqu'elle reçoit les Philosophes chez elle, sous l'influence de d'Alembert qu'elle défend ardemment. Mme Necker est très croyante, et la pensée des Philosophes la choque. Cependant elle les reçoit, et chez elle aussi on discute Philosophie. On ne peut dire non plus que Mme Geoffrin soit passionnée par la pensée nouvelle, mais elle aime favoriser les intellectuels et les artistes, et la voilà donc, bon gré, mal gré, entraînée dans la mêlée philosophique. On voit donc que même celles qui émettent quelques réserves ou même de franches réticences à l'endroit de la philosophie des Lumières contribuent à sa diffusion, d'abord parce qu'elle reçoivent les Philosophes chez elles, ensuite parce que rien n'est plus funeste que l'indifférence, et qu'il vaut mieux discuter les idées de Philosophes que n'en point parler.

À côté des tièdes ou des réticentes, il y a celles, au contraire, qui vont combattre ardemment pour les Philosophes, et dont le rôle a été si déterminant que nous allons y revenir: Mme d'Aiguillon, Mme d'Epinay, Mlle de Lespinasse.

On peut voir aussi, outre ces différences qui tiennent au tempérament des maîtresses de maison, des différences qui tiennent à l'évolution des moeurs et du goût, au triomphe progressif des Lumières. Les Goncourt distinguent trois âges des salons: le salon de 1730, celui de 1760 , celui de 1780 . Dans le salon de 1730 , 'le monde est encore en famille. C'est une assemblée intime, un plaisir qui a l'apaisement et l'heureuse tranquillité d'un lendemain de bal .... La noblesse vient seulement de "s'enversailler" et l'on trouverait dans ce salon bien clos et dans ce passe-temps d'hiver un souvenir de la vie de château.' Le salon de 1760 est 'tout brillant, tout bruissant .... C'est le plaisir dans sa vivacité, c'est le bal. Le tambourin, la flûte, la basse et le violon jettent leurs notes mariées du haut d'une estrade.' Le salon de l'époque Louis XVI est différent: ' $C$ 'est encore la société, mais ce n'est plus le plaisir. Il y a déjà, dans ce salon, l'air de 1788 et 1789; la causerie y prend des attitudes de dissertation. ${ }^{\prime 9}$ Ainsi les Goncourt décrivent-ils les trois styles des salons, en s'inspirant de trois gravures tirées de tableaux: L'Hiver de Lancret, Le Bal Paré de Saint-Aubin enfin l'Assemblée au salon de Lavreince. Évidemment, il y a là une simplification, et les Goncourt ne manquent pas d'éprouver quelque plaisir à être à leur tour peintre ou graveur. On aimerait voir les choses de plus près, et dans les perspectives qui sont ici les nôtres.

Il est vrai que la première génération des salons est plus littéraire que philosophique. C'est la littérature qui est en vedette chez la duchesse du Maine, chez Mme de Lambert ou Mme de Tencin. Ainsi dans le salon, de très bon ton, de Mme de Lambert où l'on trouve La Motte Houdart, des femmes telles que Mmes de Caumont, de Murat, 
de Caylus et la grande érudite qu'est Mme Dacier. (Cependant la séparation entre littérature et philosophie est déjà en train d'être remise en cause, et la personnalité de Fontenelle qui est très présent chez Mme de Lambert est bien symbolique de cette remise en cause.) Le jeune Voltaire dont l'esprit pétille chez la duchesse du Maine, est alors surtout littérateur. C'est le poète que l'on apprécie en lui, non pas l'auteur des Lettres philosophiques.

Les femmes vont assurer le triomphe des Philosophes dans les Salons du milieu du siècle, contemporains de la lutte et de la victoire de l'Encyclopédie. La scission du salon de Mme du Deffand est certes la conséquence de la vie personnelle de deux femmes, elle n'en est pas moins aussi un signe de l'évolution de leur rôle dans la propagande philosophique. Mlle de Lespinasse lorsqu'elle quitte son amie, emmène avec elle les éléments les plus gagnés au parti philosophique. Mais là encore, il ne faut pas simplifier à l'excès et Mme du Deffand a toujours été la protectrice de d'Alembert, même s'il y eut quelques orages dans leurs relations. ${ }^{10}$ Le salon de Mme Geoffrin est le lieu de ralliement du parti philosophique et s'y retrouvent Diderot, d'Alembert, Condorcet, Morellet, Thomas. Dans les salons de la fin de l'Ancien Régime, tel celui de Necker 'se fait le lien entre les derniers encyclopédistes et les hommes de la Révolution. ${ }^{\prime 11}$

On aimerait pouvoir analyser avec plus de précision le rôle exact des femmes. Il ne se limite pas à celui, important déjà, de maîtresse de maison. Ces femmes qui étaient souvent fort riches et pouvaient assez librement disposer de leurs biens ont été des mécènes. Mme de Tencin a fait beaucoup pour Marmontel, jeune provincial assez démuni quand il arrive à Paris. Mme du Deffand a protégé d'Alembert, alors qu'il était tout à fait inconnu, Morellet dans l'Éloge de Mme Geoffrin évoque la délicate attention avec laquelle elle complétait le mobilier des écrivains à qui elle rendait visite. L'étude des testaments est intéressante et montre qu'à plusieurs reprises ces femmes firent des legs à des écrivains. Elles ont souvent aidé puissamment à l'intégration dans le monde des lettres de jeunes écrivains qui, sans elles, ne seraient pas sortis de cette Bohême littéraire si bien analysée par R. Darnton. ${ }^{12}$

On sait aussi à quel point le rôle des femmes a été important dans les élection académiques qui alors se préparaient dans les salons. Le duc de Luyne affirme avec raison que 'les femmes sollicitent beaucoup dans les cas d'élection à l'Académie.' On a pu certes leur reprocher d'avoir parfois imposé des personnages sans grand intérêt, ainsi ce M. de Bissy, amant de Mme de Luxembourg, mais il n'est que justice de rappeler que leur choix fut souvent très heureux et contribua grandement à la transformation des Académies et au triomphe des Lumières dans ses assemblées. Mme de Lambert prépara l'élection de Montesquieu; Mme de 
Tencin fit celle de Marivaux; et Mme du Deffand, non sans difficultés d'ailleurs, fit élire d'Alembert. À partir de ce moment-là les Académies sont gagnées définitivement aux Lumières, et d'Alembert a, à son tour, un rôle déterminant dans le choix des académiciens.

Le rôle des femmes et des salons, si important soit-il, n'est-il que celui d'intermédiaire? J'aimerais montrer qu'elles ont modelé en profondeur l'esprit des Lumières qui n'eût pas été le même s'il n'avait eu ce lieu d'expression que furent les salons. On a beaucoup parlé à notre époque d'interdisciplinarité, on essaie, avec un succès inégal, de créer des rencontres, des colloques, des revues où cette interdisciplinarité se pratique. Peut-être le problème fondamental de l'interdisciplinarité est-il finalement de trouver un lieu. Ce lieu, au XVIIIe siècle, ce fut essentiellement les salons. Chez Mme de la Poplinière qui fit tant pour le succès de Rameau, se retrouvent philosophes et artistes et cette collaboration est une source d'inspiration, un enrichissement réciproque. La présence des femmes au sein des Lumières, leur rôle de diffuseur aura profondément marqué l'expression même de cette philosophie. Ce désir d'être compréhensible par tous qui aboutit à une littérature nouvelle aussi bien au niveau des formes que du style, c'est avant tout le désir d'être compris des femmes dont la formation intellectuelle de base n'est pas suffisante. Bien caractéristique par exemple, le choix de la forme épistolaire, préférée systématiquement au traité; le femme est traditionnellement reine dans le domaine épistolaire: la figure de Mme de Sévigné est symbolique de cette suprématie. Écrire par lettres, c'est en grande partie écrire pour les femmes. Cette merveilleuse clarté de l'exposé qui caractérise si bien Voltaire quand il expose le newtonisme et la philosophie d'outre-Manche, la non moins grande clarté avec laquelle Montesquieu expose ses idées politiques viennent bien de cela: il faut pouvoir être lu par ces femmes qui font les célébrités. La vulgarisation à une époque où le peuple est illettré, c'est essentiellement la féminisation.

Ainsi s'explique aussi le caractère très largement oral de la littérature de cette époque. Les idées avant d'être écrites ont été parlées. La lettre est une transposition de la parole; on aime la forme du dialogue. Ainsi les audacieuses théories du Rêve de d'Alembert sont exposées devant Julie de Lespinasse qui ne manque pas de demander des éclaircissements. La présence des femmes donne donc son caractère propre à l'exposé philosophique des Lumières; elles contribuent à cette admirable clarté, à la fluidité de la langue.

Mais il serait terriblement restrictif de limiter ainsi l'influence des femmes à ce qui se passe dans les salons. Elles ont aussi d'autres sphères d'action, plus éclatantes ou plus secrètes. Le rôle politique des femmes a été capital. Évidemment, ce qui choque notre féminisme 
moderne, c'est qu'il s'agit toujours d'un rôle indirect, en ce sens qu'il n'est pas pensable qu'une femme, par exemple, soit ministre. Néanmoins il ne faut pas oublier que la politique au XVIIle siècle se fait largement par des voies indirectes, qu'elle ne peut même se faire autrement à une époque qui ignore le suffrage universel. Les femmes ne sont donc pas plus désavantagées que la plupart des hommes. Mais quand on étudie le rôle politique des femmes au XVIIIe siècle, on a trop tendance à se borner à évoquer les humeurs fantasques d'une maîtresse et à déplorer les conséquences de ses caprices sur la vie politique de l'époque. Or les femmes qui avaient la dimension d'être des 'hommes d'état' étaient loin de s'abandonner à de futiles caprices. Que l'on songe à Mme de Tencin, à la ténacité, la lucidité aussi qui la caractérise, dans toute l'ardeur qu'elle mettra à faire la carrière politique de son frère. Mme de Pompadour est une tête froide, contrairement à ce qu'en a fait l'imagerie populaire. Elle a courageusement, énergiquement défendu les Philosophes, et sans elle, l'Encyclopédie n'aurait pu triompher de toutes les difficultés auxquelles la grande entreprise philosophique eut à se heurter.

Le rôle de Mme de Pompadour est bien connu, il faudrait peut-être pour avoir une idée plus complète de l'ensemble de la question, poursuivre des recherches dans des zones beaucoup plus méconnues et ne pas s'en tenir à la brillante scène de la Cour. Les femmes sont présentes et actives aussi dans les sociétés secrètes, et c'est au XVIIIe siècle qu'elles obtiennent de pénétrer dans la Maçonnerie jusque là étroitement masculine. Bien caractéristique cette phrase de l'auteur anonyme de $\mathrm{La}$ Maçonnerie des Dames: 'Il était réservé aux jours heureux qui nous éclairent de réunir les deux sexes dans nos loges. ${ }^{13}$ Un lien est donc nettement marqué entre le progrès des Lumières et la présence des femmes. Les femmes ne furent d'ailleurs pas admises dans les anciennes loges, mais furent créées des loges d'adoption où elles figuraient à côté des hommes, il semble à partir de 1772 . Alors se multiplient les ouvrages qui expliquent les modalités de leur intégration. ${ }^{14}$ Les loges d'adoption sont envahies par les femmes. Les femmes les plus brillantes de la Cour se firent admettre dans la loge de la Candeur ou dans la loge des Neuf Soeurs. Mmes de Choiseul-Gouffier, de Rochechouart, de Lomémie, de Genlis présentées par la duchesse de Bourbon's s'imposent dans la loge de la Candeur. Mme Helvétius préside la loge des Neuf Soeurs, et Mme de Lamballe celle du Contrat social. Des femmes ont même créé à leur tour des loges, ainsi la comtesse Potocska fonde celle des chevaliers de Notre-Dame-de la Persévérance. Quoique le phénomène soit largement parisien, il ne l'est pas exclusivement. On signale en province quelques loges où les femmes sont actives: celle de la Sincérité à Besançon, celle de la Parfaite amitié à Toulouse, et 
à Confolens, celle de la Parfaite union. Le rôle politique des Loges a été si souvent un sujet de controverses que nous ne voudrions pas y revenir. D'après ce que nous pouvons savoir de l'activité des femmes dans les Loges, elle se ramène souvent à des oeuvres de bienfaisance mais elles contribuent à la laïcisation de l'idée de charité, à sa transformation en la notion d'humanitarisme, si caractéristique des Lumières.

Les communautés religieuses sont parfois des bastions de la pensée rétrograde, certes, mais ce n'est pas une loi universelle. Dans la mesure où la religieuse est relativement libre par rapport à la femme mariée, elle garde souvent plus d'indépendance de pensée que sa contemporaine écrasée par la fatigue et les dangers des maternités. D'autre part le rôle des couvents est fondamental dans l'éducation des filles. Certes, les Philosophes ont dénoncé énergiquement l'obscurantisme qui sévit dans cette éducation, et il serait vraiment trop paradoxal de vouloir soutenir que les religieuses ont contribué au progrès des $\mathrm{Lu}$ mières. Il faut cependant rappeler qu'aucune organisation n'est prévue pour l'instruction des filles du peuple. C'est grâce aux religieuses que ces filles sortent de l'ignorance totale, qu'elles apprennent à lire et à écrire, même si, par ailleurs, leurs professeurs en profitent pour leur communiquer une idéologie qui nous semble aux antipodes des Lumières. Encore faudrait-il connaître mieux le détail de ces existences obscures de religieuses et l'on n'a pas encore découvert de testament de soeur Meslier.

En tout cas, premières victimes de l'obscurantisme des vocations forcées, elles sont prêtes à faire front commun avec les Philosophes à ce sujet. Témoin cette jeune religieuse qui écrit à Louis $X V$ pour s'étonner que les lois aient fixé un âge minimal avant lequel une femme ne peut passer un contrat, mais n'aient pas prévu d'âge minimal pour prononcer des voeux. ${ }^{15}$ Lorsque l'on étudie le relâchement des couvents féminins au XVIIIe siècle, c'est toujours sous son aspect le plus superficiel et le plus mondain, pour évoquer par exemple le luxe, les raffinements des chanoinesses de Remiremont; on néglige que chez les plus intelligentes, ce n'est pas tant le luxe qu'on réclame que les Lumières. Ainsi les bénedictines d'Almenèches font certes venir de Paris des onguents, mais aussi elles s'abonnent au Mercure de France. ${ }^{16}$ La Religieuse de Diderot connaît des communautés d'esprit bien divers: on a retenu surtout le saphisme qui règne dans un de ces couvents, ne faudrait-il pas aussi rappeler que l'on y joue de cet instrument tant aimé des Lumières: le clavecin, et que l'on y suit la vogue de la musique italienne, vogue à laquelle les Philosophes ont beaucoup contribué.

Les supérieures ne parviennent plus à maintenir leur autorité; certes, ces révoltes sont souvent futiles; mais ne sont-elles pas aussi le signe 
de cette remise en cause du principe d'autorité qu'au même moment attaquent les écrivains. Et Stendhal lorsqu'il imaginera L'abbesse de Castro, montrera bien comment une révolte en apparence futile, la demande d'une femme de chambre supplémentaire, n'est qu'un prétexte pour manifester une volonté d'indépendance. Ces révoltes de religieuses seraient tout particulièrement intéressantes à étudier. La société monastique est certes un microcosme, mais où se jouent tous les mécanismes du pouvoir, et la supérieure figure l'image du 'despotisme' que n'est certes pas souvent 'éclairé.' Parmi les couvents dont les révoltes sont célèbres, citons, outre l'abbaye d'Almenèches (Essay, Normandie), le couvent de Saint-Laurent de Comminges. ${ }^{17}$ On aurait tort d'invoquer le fait qu'elles demandent à rester en communauté au moment de la Révolution: c'est souvent peur de l'inconnu, manque de ressources matérielles essentiellement. Cependant beaucoup acceptèrent avec plaisir que la liberté leur soit rendue.

Parce que la religion est largement supportée et portée par les femmes, c'est là aussi le domaine où elles peuvent marquer leur indépendance d'esprit, et l'on a trop eu tendance à y voir au contraire, le domaine où elles sont la proie de l'obscurantisme. Sans nier, ce qui est évident, que la religion a le plus souvent travaillé à freiner le travail des Philosophes, il faudrait rappeler cependant que le rapport Foi/Lumières n'est pas aussi simple qu'on l'a trop souvent cru, et que contester des analyses simplistes et réductrices, ce n'est pas seulement apporter des éléments nouveaux à l'histoire de la pensée religieuse et philosophique, c'est aussi, du même coup, repenser le rôle des femmes au XVIIIe siècle. L'articulation entre Foi et Lumières n'est pas la même d'ailleurs en France et à l'étranger où la pensée philosophique est loin d'être toujours antireligieuse, ainsi en Pologne ou dans les pays protestants. Le piétisme gagné à la fois aux Lumières et à l'illuminisme est un courant de pensée et de sensibilité dont on ne cesse de découvrir l'importance et dans lequel les femmes ont joué un rôle déterminant. Pour nous en tenir cependant à un domaine que nous connaissons mieux, la France, le rôle des femmes a été capital dans la diffusion et les progrès du quiétisme qui, à sa naissance même, avait été l'oeuvre d'une femme, Mme Guyon. La religion de Mme de Warens a été bien étudiée; mais il y eut beaucoup de Mme de Warens qui n'eurent pas la chance d'avoir un Jean-Jacques Rousseau pour parler d'elles. Le fénelonisme trouva d'ardentes propagandistes parmi les femmes; or on sait, l'importance de Fénelon dans la pensée des Lumières, du moins dans le versant déiste de cette pensée.

La religion a abruti les femmes? Elle leur a permis aussi d'exercer leur réflexion et de manifester leur esprit de révolte dans le seul domaine qu'on leur laissait. Ainsi, aux antipodes du courant quiétiste, 
dans les querelles du jansénisme. Là encore il reste beaucoup à faire pour éclaircir la question des rapports ambigus du jansénisme et des Lumières, et le bel ouvrage de M. Imbert sur Stendhal où sont montrées les articulations entre l'idéologie et le jansénisme devrait servir d'exemple, si l'on ne s'obstinait à vouloir ignorer la réflexion religieuse des Philosophes. Pour en venir à notre époque, il est bien évident que les Philosophes n'avaient aucun goût pour l'ascétisme, et que le jansénisme leur apparaissait comme une forme particulièrement sinistre d'obscurantisme. Cependant, dans la mesure où il était un des points chauds de la contestation du pouvoir royal, ils ont eu parfois quelque sympathie pour ces révoltés de la bulle Unigenitus. Or dans la résistance janséniste contre le pouvoir, les femmes ont eu une place fort importante que les propos de Montesquieu dans les Lettres persanes reflètent: 'Les femmes, écrit-il dans la XXIVe des Lettres persanes, sont les motrices de toute la révolte qui divise la Cour, tout le royaume et toutes les familles'; ce que confirme Barbier en 1728: 'Le parti janséniste est fort et remuant comme un diable. Les femmes, femmelettes et jusqu'aux femmes de chambre s'y feraient hâcher. ${ }^{\prime 18}$ Les femmes écrivent en faveur du jansénisme - telles Mme de Jaucourt ou Mme Perrier qui dirigent des imprimeries clandestines, et dans la dispute entre le cardinal de Tencin et Soanen, évêque de Senez et janséniste notoire, prennent parti pour ce dernier. Barbier raconte qu'on trouva un placard janséniste dans l'église Saint-Séverin et que ce placard avait été affiché par une femme. Si j'insiste, c'est pour montrer que dans l'affaire janséniste, les femmes ne se limitent pas à l'hystérie et aux convulsions. Certaines ont eu un rôle véritablement politique et en contestant l'autorité royale et papale, elles rejoignent la contestation des Lumières. La scène où le héros de Voltaire se retrouve en prison avec un janséniste prend figure de symbole. Si différentes que soient l'idéologie janséniste et celle des Philosophes, ils se retrouvent dans le même élan contestataire.

Un autre domaine où la résistance des femmes fut grande, c'est le protestantisme. Et là la cause commune avec les Lumières est encore beaucoup plus évidente. Les femmes étaient nombreuses dans les assemblées du Désert.

En 1754, moment où recommencent les persécutions violentes une infinité de femmes se trouvent enfermées dans les couvents ou les hôpitaux du Languedoc (Tarbes, Cahors, Montauban, Carcassonne) du Dauphiné (Die, Vienne) et de la Saintonge. ${ }^{19}$

C'est aussi le moment où l'Encyclopédie se heurte à l'hostilité du pouvoir. L'affaire de la thèse de l'abbé de Prades le prouve. Il y avait pire 
que le couvent, et Marie Durand passa trente-sept ans de sa vie dans la tour de Constance. Même si tous les cas ne furent pas aussi dramatiques et s'il y eut des résistances plus passives, les femmes protestantes étaient intéressées au plus haut degré à la lutte que menèrent les Philosophes pour la reconnaissance de la validité du mariage des protestants, car le concubinage sous l'Ancien Régime était plus gênant pour elles que pour les hommes. Certaines femmes protestantes s'exilèrent en Hollande comme les Philosophes persécutés.

III

On voit donc combien, sur beaucoup de points, les femmes pouvaient faire cause commune avec les Philosophes, et cela à des niveaux fort divers, dans l'atmosphère charmante d'un salon élégant, comme dans l'obscurité de la vie quotidienne, ou même dans les horreurs de la persécution. Mais nous voulons aller plus loin; les femmes n'ont pas été de simples échos des Lumières, si elles ont joué un rôle dans la diffusion de la pensée des Philosophes (et pas seulement dans les Salons: il se peut même que ce travail obscur de femmes inconnues en révolte contre le pouvoir ait été tout aussi important pour le progrès des Lumières en France, que le rôle de tel brillant salon dans une élection académique), elles ont travaillé, elles ont créé de conserve avec leurs homologues masculins, et ce sont maintenant ces pionnières de la recherche et de la littérature, leur rôle dans la vie intellectuelle du XVIIIe siècle que nous voudrions évoquer de façon forcément sommaire. Nous laissons de côté la question si importante des femmes journalistes, parce qu'une communication leur est consacrée aujourd'hui même.

Un des aspects les plus intéressants du mouvement philosophique consiste dans l'activité qu'il déploya dans le domaine scientifique. La passion des Philosophes pour les sciences expérimentales, l'importance de d'Alembert, à la fois grand mathématicien et co-directeur de l'Encyclopédie, du moins à ses débuts, sont des faits bien connus. Mais lorsqu'il s'agit de femmes, la critique traditionnelle a tendance à évoquer avec un sourire amusé et quelque peu condescendant, ces savants qui passaient des journées dans leur laboratoire. Et cela conformément à ce cliché (qui d'ailleurs est davantage le produit du XIXe et du début du XXe siècle) que les femmes ne sont pas douées pour les sciences, que les filles sont toujours 'littéraires.' Mme du Châtelet est si connue, l'ouvrage récent d'E. Badinter a tellement attiré l'attention sur Émilie, que l'on a quelque scrupule à rappeler ce nom, pourtant indispensable. ${ }^{20}$ Sa formation scientifique est des plus sérieuses: elle a été l'élève du grand mathématicien Clairault et de Maupertuis. Elle travaille avec 
un acharnement qui a frappé tous les contemporains. Le Président Hainault quand il passe à Cirey et voit Voltaire et Mme du Châtelet, note: 'L'un fait des vers de son côté, l'autre des triangles. ${ }^{21}$ La correspondance qu'entretient Mme du Châtelet avec Maupertuis nous prouve combien son travail était assidu; elle se plaint, le 20 juin 1739, 'je travaille beaucoup, et je n'avance guère. Imaginez-vous que je suis obligée de souper souvent en ville, je me lève tous les jours à six heures au plus tard pour étudier.'22 Elle est persuadée que l'étude est plus nécessaire aux femmes qu'aux hommes, contrairement au préjugé, et elle écrit dans le Discours sur le bonheur:

Il est certain que l'amour de l'étude est bien moins nécessaire au bonheur des hommes qu'à celui des femmes ... les femmes sont exclues, par leur état, de toute espèce de gloire, et quand par hasard, il s'en trouve quelqu'une qui est née avec une âme assez élevée, il ne lui reste que l'étude pour la consoler de toutes les exclusions et de toutes les dépendances auxquelles elle se trouve condamnée par état. ${ }^{23}$

La préface à la traduction de la Fable des abeilles est une énergique revendication féministe: 'Je sens tout le poids du préjugé qui nous exclut, si universellement des sciences, et c'est une des contradictions de ce monde, qui m'a toujours le plus étonnée, car il y a de grands pays, dont la loi nous permet de régler la destinée, mais, il n'y en a point où nous soyions élevées à penser.' C'est par la faute de leur éducation ou plutôt de leur absence d'éducation, que les femmes ne sont pas créatrices:

Qu'on fasse un peu réflexion pourquoi depuis tant de siècles jamais une bonne tragédie, un bon poème, une histoire estimée, un beau tableau, un bon livre de physique n'est sorti de la main des femmes? .... Je laisse aux naturalistes à en chercher une [cause] physique, mais jusqu'à ce qu'ils l'aient trouvée, les femmes seront en droit de réclamer contre leur éducation. Pour moi j'avoue que si j'étais roi, je voudrais faire cette expérience de physique. Je réformerais un abus qui retranche pour ainsi dire la moitié du genre humain. Je ferais participer les femmes à tous les droits de l'humanité, et surtout à ceux de l'esprit. ${ }^{24}$

Sa propre expérience est évoquée à l'appui de son affirmation: 'Je suis persuadée que bien des femmes ignorent leurs talents, par le vice de leur éducation, ou les enfouissent par préjugé, et faute de courage dans l'esprit.' ${ }^{25}$ On voit ici comment la revendication s'inscrit absolument dans la Philosophie des Lumières: volonté de détruire les préjugés, désir de leur opposer l'expérience, appel au 'courage de l'esprit,' c'est le 'sapere aude' de Kant; enfin nécessité de commencer la réforme de 
l'homme et de la femme par une transformation de l'éducation dont les Lumières sont persuadées qu'elle peut tout, ou presque et affirmation de l'universalité des droits pour toute l'humanité.

La figure de Mme du Châtelet demeure exemplaire par cette passion de savoir et de communiquer son savoir par l'écrit. À la veille de mourir, victime du sort qui fut celui de tant de femmes, elle écrit à Claude Sallier ce qui fut sa dernière lettre, pour lui confier la sauvegarde de ses manuscrits:

J'use de la liberté que vous m'avez donnée, monsieur, de remettre entre vos mains des manuscrits que j'ai grand intérêt qu'ils restent après moi. J'espère bien que je vous remercierai de ce service et que mes couches, dont je n'attends que le moment, ne seront pas aussi funestes que je le crains. Je vous supplierai de vouloir bien mettre un numéro à ces manuscrits et les faire enregistrer afin qu'ils ne soient pas perdus. ${ }^{26}$

Les manuscrits furent sauvés, mais les couches furent 'funestes.'

L'oeuvre de Madame du Châtelet est difficile à évaluer, mais ni plus ni moins que celle des savants de son époque. Il est bien évident que les sciences sont le domaine dans lequel les écrits vieillissent le plus vite. Mais il y a au moins une certitude: c'est que Mme du Châtelet est bien l'auteur de ses oeuvres. Dénonçons une fois pour toutes ce préjugé qui veut que, dès qu'une femme écrit quelque chose de remarquable, $c^{\prime}$ est en fait un homme qui en est l'auteur. L'éditeur de sa correspondance, Besterman a pris énergiquement, et preuves en main, la défense de Mme du Châtelet: 'un sceptique pourra toujours prétendre que les mérites de la Dissertation sur la nature $d u$ feu, les Institutions et le grand commentaire sur Newton, sont redevables à Maupertuis, König et Clairault. Il nous semble impossible de maintenir ce point de vue après la lecture de nombreux passages jusqu'à présent inédits des lettres à Maupertuis, et des nouvelles épitres à Bernouilli et Jurin.' En effet, 'il ne faut pas oublier que beaucoup de ces lettres étaient écrites précisément à ceux que l'on prétend avoir été ses nègres. Toutes ces discussions auraient été bien sottes adressées à des personnes qui l'en auraient cru incapable. ${ }^{27}$ Il faut donc bien reconnaître à Mme du Châtelet la gloire d'avoir manifesté cet idéal scientifique qui est celui des Lumières. Elle possède 'un esprit scientifique qui a bien exactement conçu les principes fondamentaux de la physique et de la méthodologie scientifique sur laquelle toute vérité est basée. ${ }^{28}$ Son oeuvre est considérable: Dissertation sur la nature et la progation du feu (Prault, 1744), un Essai sur l'optique, dont il ne reste qu'un chapitre, conservé à Leningrad, Les Institutions de physique (Prault, 1740), sa traduction des Principes de Newton: Principes mathématiques de la philosophie naturelle par Newton 
(Desaint et Saillant, 1759), donc posthume. Sans oublier diverses lettres de polémique, des réponses à des attaques. Elle participe de tout ce courant philosophique des Lumières qui opte pour Newton contre la tradition cartésienne des tourbillons.

Chez elle la passion de la physique s'accompagne d'une passion de la philosophie et de la métaphysique. Et dans ce domaine, sa pensée est encore plus mal connue. Voltaire en septembre 1749 écrit à d'Argental: 'Je viens de relire les matériaux immenses de métaphysique que Mme du Châtelet avait assemblés avec une patience et une sagacité qui m'effraient ... c'était le génie de Leibniz avec de la sensibilité.' Elle oeuvre aussi dans le sens de tout un courant des Lumières, en soumettant la Bible à une exégèse sévère, et en optant pour un déisme naturaliste. Son Examen de la Genèse a été étudié par I.O. Wade dans Voltaire et Madame du Châtelet. On y voit une érudition étonnante, des curiosités historiques, linguistiques aussi - Mme du Châtelet est bien en cela une femme des Lumières, elle qui avait entrepris une Grammaire raisonnée. La métaphysique de Newton l'intéresse autant que sa physique, même si finalement elle lui préfère, en ce domaine Leibniz; elle traduit la Fable des Abeilles de Mandeville. Dans les Institutions de Physique, elle entend secouer la paresse de son siècle à l'endroit de la métaphysique: 'Les obscurités dont quelques-unes des parties de la Métaphysique sont encore couvertes servent de prétexte à la paresse de la plupart des hommes pour ne la point étudier' (13). Certes, il ne faut pas abandonner en ce domaine les règles de la rigueur scientifique. Il faut étudier la physique avant la métaphysique: 'Souvenezvous, mon fils, dans toutes vos études, que l'expérience est le bâton que la nature a donné à nous autres aveugles, pour nous conduire dans nos recherches' (10). C'est cette observation du monde qui la conduit à une métaphysique leibnizienne, même si elle convient qu'il y a là un raisonnement logique qui n'est plus de l'ordre de l'expérience:

notre impuissance (à voir la totalité de l'univers) ne peut nous faire douter que l'Intelligence suprême n'ait choisi le meilleur des mondes pour lui donner l'existence; car l'Être nécessaire qui se suffit à lui-même, et qui n'a besoin d'aucune chose hors de lui, n'a pu se proposer d'autres fins dans la Création de cet univers, que de communiquer une partie de ses perfections à ses Créatures et de faire un ouvrage digne de lui, puisqu'il se serait manqué à lui-même et qu'il aurait dérogé à ses perfections, s'il avait produit un monde indigne de sa sagesse.

Voltaire ne suivait pas Mme du Châtelet dans cet optimisme leibnizien, mais elle n'en est pas moins très caractéristique de tout un courant des Lumières spiritualiste et déiste, quand elle affirme: 'L'Étude de la na- 
ture nous élève à la connaissance de l'Être suprême' (38). Si Voltaire n'était pas leibnizien, il fut constamment déiste.

Mais si la figure d'Émilie domine dans cette évocation des Lumières scientifiques et féminines, il serait injuste de passer sous silence des noms moins célèbres, provinciaux, ainsi celui de Mme Lepaute qui présente à l'Académie de Béziers des mémoires d'astronomie, et dont la Bibliothèque Nationale possède la savante Explication de la carte qui représente le passage de l'ombre de la lune au travers de l'éclipse du soleil du 16 avril $1765 .{ }^{29}$ Si Algarotti publie un Newtonisme pour les dames, que Madame du Châtelet juge d'ailleurs, malgré son amitié, trop 'frivole, ${ }^{\prime 30}$ ce n'est pas pour faire sourire, mais parce qu'il était conscient du grand intérêt que les femmes portaient à l'astronomie; et l'on a tort de perpétuer à l'égard de ces recherches scientifiques des femmes la lourde ironie de Chrysale devant 'cette grande lunette à faire peur aux gens.'

Il est un autre domaine scientifique où leur rôle fut important dans la lutte contre les ténèbres des préjugés: la médecine. Et d'abord ce secteur encore si archaïque au XVIIle siècle, celui de la parturition. Mme du Coudray fut un véritable apôtre d'une rationalisation des méthodes de gynécologie; elle allait de ville en ville enseigner les sages-femmes, et elle a écrit un fort intéressant Abrégé de l'art des accouchements. Elle devrait figurer en bonne place dans une Histoire des Lumières. L'inoculation qui fut aussi un des thèmes de la bataille philosophique, a eu beaucoup d'adeptes parmi les femmes, et l'inoculation n'avait-elle pas justement été lancée par une femme, lady Montagu? Les grandes dames de la Cour, Mlles de Chartes, de Vaucanson et de Senectère, la marquise de Villeroi, la comtesse de Forcalquier se firent inoculer; ces exemples venus de haut furent tout aussi importants que les écrits des Philosophes dans cette 'traque' de la variole. Enfin les principes $\mathrm{d}$ 'hygiène que demandait ce médecin des Lumières que fut Tronchin, leur parurent avoir la force de l'évidence, et là aussi elles font cause commune avec les Philosophes.

À mi-chemin entre la science et l'histoire, notons le curieux Discours sur la naissance et les progrès de la chimie, oeuvre de la présidente d'Arconville qui contient de vues intéressantes sur la préhistoire et sur l'origine des techniques industrielles. ${ }^{31}$ Les réflexions politiques ne font pas peur aux femmes qui entretiennent un dialogue parfois critique avec les Philosophes, ainsi Mme Belot écrit des Réflexions d'une provinciale sur le discours sur l'inégalité32 de Rousseau, et Mme Dupin des Observations sur l'esprit des lois. C'est dans cette lignée que s'inscrit, en les dépassant, le jeune talent de Mme de Staël lorsqu'elle écrit en 1788 les Lettres sur les ouvrages et le caractère de Rousseau.

Les oeuvres historiques, les vies de grands hommes, les 'éloges' sont aussi des genres où s'illustrent les femmes. Mais la personnalité qui 
domine, dans le domaine de la critique littéraire, c'est Mme Dacier, traductrice de l'lliade et de l'Odyssée, l'ardente championne des Anciens; et il est un peu simpliste de penser que ce sont forcément les Modernes qui annoncent les Lumières et les Anciens qui défendent l'obscurantisme. Pour ce qui est du féminisme, la personnalité de Mme Dacier suffit à elle seule à répartir le discours féministe entre Anciens et Modernes. Cette grande érudite dont la science est tellement au-dessus de ses adversaires, par exemple d'un La Motte, mériterait d'être beaucoup mieux connue. ${ }^{33}$

Si l'on en vient, dans ce rapide panorama, à la création littéraire, non sans avoir rappelé l'existence des femmes peintres, de Mme VigéeLebrun, l'importance des musiciennes, qu'on dit plus interprètes que compositeurs (encore faudrait-il voir les choses de plus près, n'y aurait-il pas une Élizabeth de la Guerre à la génération des Lumières?) on est amené à constater malheureusement l'existence de genres où elles excellent et d'autres où elles ne se sont pas du tout illustrées. Cependant il ne semble pas que les hommes du XVIIIe siècle aient eu les préjugés de ceux du XXe à l'endroit des femmes dramaturges. Ils ont fait bon accueil à la Cénie de Mme de Graffigny (1755), et si sa Fille $d^{\prime}$ Aristide (1758) tombe, ce n'est pas parce qu'elle est l'oeuvre d'une femme, mais parce qu'elle est médiocre, Mme du Bocage connut aussi un certain succès avec ses Amazones. ${ }^{34} \mathrm{Il}$ faudrait aussi évoquer la personnalité de Mme Favart. ${ }^{35}$ Elle fait cause commune avec Diderot et les Philosophes pour réclamer une simplification des costumes au théâtre et pour demander plus de couleur locale, plus de vérité, par exemple dans les costumes antiques, l'abandon des 'paniers.' Mlle de Saint-Léger, comme Lesage, comme Beaumarchais écrit pour le théâtre de la Foire, une pièce assez réussie, les Deux soeurs. ${ }^{36}$ Notons aussi l'activité dramatique des femmes dans les salons.

Il est bien vrai cependant que l'originalité des femmes du XVIIIe siècle éclate davantage dans ce que l'on pourrait appeler les écritures du Moi et dans le roman qui souvent s'apparente à ce type d'écriture. On peut même dire, sans exagération, qu'elles ont grandement travaillé à cette explosion de l'écriture du moi à la fin du XVIIIe siècle qui peut être considérée comme la conséquence du progrès des Lumières dans leur revendication des droits de l'individu. On les voit passer tout naturellement des chroniques de la vie du temps à l'autobiographie. Les Mémoires de Mme de Staal-Delaunay ${ }^{37}$ appartiennent davantage à ce premier registre, tandis que les 'pseudo'-mémoires de Mme d'Épinay relèvent déjà du second.$^{38}$ Les Goncourt ont eu raison d'attirer l'attention sur l'importance de cet ouvrage. Le registre autobiographique est exploité aussi grâce à l'utilisation de ce genre où la suprématie des femmes avait été de longue date reconnue: les lettres. Ces femmes dont 
nous avons évoqué tout à l'heure le rôle auprès des Philosophes en tant qu'elles leur ouvraient leur salons, il faut maintenant dire qu'elles sont, du moins certaines d'entre elles, de véritables écrivains. Les Lettres de Julie de Lespinasse ${ }^{39}$ celles de Mme du Deffand ${ }^{40}{ }^{\prime}$ 'intéressent pas seulement comme témoignage sur d'Alembert, sur les philosophes, elles ont une valeur intrinsèque. On y lit l'expression des passions. Quel dommage que nous n'ayons plus les Lettres de Sophie Volland - les quelques citations qu'en donne Diderot nous les font regretter davantage. Mais nous avons les Lettres de Mme d'Épinay, ${ }^{41}$ en proie à une passion souvent mal récompensée pour Grimm; nous avons surtout les lettres d'Émilie du Châtelet que son amour pour Voltaire dévore, rend littéralement malade, que sa passion pour SaintLambert fera souffrir encore jusqu'à la mort. Ces femmes contribuent à réaliser ce passage si important des Lumières au romantisme, passage qui s'opère bien entendu aussi chez un Diderot ou un Rousseau.

L'écrit moral devient souvent chez les femmes une forme de l'écriture du moi. La pédagogie et la confidence s'y rejoignent. Les oeuvres de Mme de Lambert, Avis d'une mère à sa fille, Réflexion sur les femmes, De l'Amitié sont largement autobiographiques. ${ }^{42}$ Davantage encore ce pur chef-d'oeuvre qu'est le Discours sur le bonheur de Mme du Châtelet. Écrit dans un moment de vide, entre la passion pour Voltaire qui s'est transmuée, non sans peine, en amitié, et la passion pour SaintLambert qui n'a pas encore éclaté, cet ouvrage traduit non tant un effort vers la sagesse qu'une profonde insatisfaction: elle amène à des confidences qui sont bien du registre de l'autobiographie. Certes l'ouvrage s'inscrit dans une 'tradition morale' de l'épicurisme moderne' pour reprendre les termes qu'emploie R. Mauzi dans sa belle préface. Mme du Châtelet reprend un certain nombre de thèmes que l'on trouve chez Pope et dans les Discours sur l'homme de Voltaire. Cependant nous touche davantage la confidence directe, celle-ci par exemple sur le bonheur qu'elle a connu avec Voltaire, et qui pour avoir été souvent citée, n'en demeure pas moins fort belle:

J'ai été heureuse pendant dix ans par l'amour de celui qui avait subjugué mon âme; et ces dix ans je les ai passés tête-à-tête avec lui, sans aucun moment de dégoût, ni de langueur. Quand l'âge, les maladies, peut-être aussi la facilité de la jouissance ont diminué son goût, j'ai été très longtemps sans m'en apercevoir; j'aimais pour deux, je passais ma vie entière avec lui, et mon coeur, exempt de soupçon, jouissait du plaisir d'aimer et de l'illusion de se croire aimé. Il est vrai que j'ai perdu cet état si heureux, et que ce n'a pas été sans qu'il m'en ait coûté bien des larmes.

Enfin le roman qui est le genre le plus florissant du XVIIIe siècle, ne serait pas ce qu'il est sans toutes ces femmes auxquelles on songe 
immédiatement, sans toutes ces oeuvres qu'on redécouvre, et qu'on réédite. La récente réédition du Comte de Comminges $^{43}$ de Mme de Tencin prouve le succès des femmes dans le roman historique. À la fin $\mathrm{du}$ XVIIe siècle, Mlle Bernard, nièce de Corneille et parente de Fontenelle aurait écrit une Elénore d'Yvrée, une Inès de Cordoue que ne sont pas dénuées d'intérêt. ${ }^{44}$

Les femmes ont beaucoup contribué à la vogue du roman par lettres, en quoi elles rejoignent Montesquieu, Richardson, Rousseau. Chez les femmes comme chez leurs confrères masculins, la lettre est utilisée dans des registres assez différents. Elle appartient au registre prétendûment exotique avec les Lettres circassiennes de Mlle Aissé ou les Lettres péruviennes de Mme de Graffigny. Mme Riccoboni emploie avec prédilection cette forme qui se prête si bien à l'analyse psychologique et à l'expression de la subjectivité. ${ }^{45}$ Le pur chef-d'oeuvre que sont les Lettres de Lausanne et sa suite Caliste de Mme de Charrière est l'aboutissement de cette tradition. Et il convient de rappeler que la Correspondance littéraire de Grimm souligna ce que ce roman avait de tout à fait exceptionnel par sa qualité et son intensité. Benjamin Constant le connaissait bien et pour cause quand il écrivit Adolphe.

Comme les Philosophes, les femmes ont su faire du roman un instrument de la diffusion des idées philosophiques. On ne connaît peutêtre pas assez l'oeuvre de Mme Robert qui se fait une apôtre de la Physiocratie dans son Voyage de milord Céton dans les sept planètes ou le nouveau Mentor. ${ }^{46}$ Mais le roman est surtout le lieu où se fait jour la revendication contre les préjugés et l'oppression masculine, ainsi dans les Lettres de Milady Juliette Catesby de Mme Riccoboni, et où s'expriment les droits de la sensibilité, vertu chère aux Philosophes. ${ }^{47}$

$\mathrm{Y}$ a-t-il une véritable spécificité du roman féminin, et cette question va nous amener à en poser une autre: y a-t-il une véritable spécificité des Lumières féminines? Le roman féminin qui a souvent pour objet de dépeindre la condition féminine vue de l'intérieur, a un aspect assez reconnaissable, quoiqu'il puisse y avoir des effets de reflets, et que Rousseau ou Marivaux soient très capables de prendre un style que nous considérons comme féminin. Pour ce qui est d'une spécificité des Lumières au féminin, les nuances doivent être encore plus nécessaires. Si beaucoup de femmes ont fait front commun avec les Philosophes, il est bien évident que dans l'ensemble des thèmes que ceux-ci ont mis en vedette, certains leur sont plus sensibles. Et d'abord ceux qui concernent la condition de la femme: la question des vocations religieuses forcées, celle du mariage imposé par la famille, la question aussi de l'éducation des enfants, de l'allaitement, du droit au bonheur. Mais leur combat n'est pas véritablement différent de celui des hommes. Il s'agit toujours de lutter pour la liberté de l'individu, pour le respect 
de ses droits fondamentaux. Et c'est peut-être parce que, du moins dans le domaine des lettres et de la philosophie, l'idéologie des Lumières va vite être dominante, qu'il n'y a pas au XVIIIe siècle un véritable mouvement féministe, comme en verront naître le XIXe et le XXe siècle. Les femmes ne font que demander que l'on applique à leurs problèmes spécifiques, les grands et beaux principes que les Philosophes réclament pour tout être humain (même si individuellement, il peuvent avoir des réflexes que nous jugerions sexistes ou racistes; il leur arrive, nous l'avons vu et c'est particulièrement sensible chez Rousseau, d'être gênés par une conception de la différence qui ne parvient pas à éliminer la notion de hiérarchie). La conséquence logique de l'application des principes des Lumières serait, comme Mme du Châtelet le dit clairement, la reconnaissance des droits de la femme autant que de ceux de l'homme.

Si les femmes n'ont pas de raison au XVIIIe siècle de faire bande à part, et de mener un combat distinct de celui des Lumières féminines, il est évident, en revanche, que les femmes ont permis au discours des Philosophes d'atteindre une véritable universalité. Par la diffusion dans les salons, certes, par l'influence qu'elles ont eue dans cette suprême intelligibilité qui caractérise nos Philosophes du XVIIIe siècle, mais aussi, tout simplement, parce que l'on ne conçoit guère qu'un discours qui vise à l'universalité, puisse exclure la moitié du genre humain. Par le fait même qu'elle s'exprime, la femme lutte contre les préjugés, et par conséquent s'inscrit dans le camp des Lumières. C'est bien ce que dit l'abbé de la Porte lorsqu'il publie en 1767 son Histoire littéraire des femmes françaises: il a entrepris de 'faire voir ce que la femme peut faire dans la carrière des lettres lorsqu'elle sait se mettre au-dessus de préjugés. ${ }^{\prime 48}$ À son tout, elle apporte aux Lumières non seulement un écho, mais la complétude même de ce concert qui ne peut être grand que si les voix aiguës se joignent aux voix plus graves. De Mme Dacier à Mme de Staël, la présence des femmes dans la pensée du XVIIIe siècle est fondamentale. Sans ces femmes, les Lumières n'auraient pas été ce qu'elles sont.

BEATRICE DIDIER

Université de Paris VIII 


\section{Notes}

1 La femme et le féminisme avant la Révolution (Leroux, 1923).

2 Ainsi les précieuses Lettres de la marquise du Châtelet, introd. et notes de Th. Besterman, 2 vol. (Genève: Institut Voltaire, 1958); Mme du Châtelet, Discours sur le bonheur, édit. R. Mauzi (Belles Lettres, 1961): Mme d'Epinay, Histoire de Madame de Montbrillant, introd. et notes de G. Roth, 3 vol. (Gallimard, 1962); Noton enfin, plus récemment, les excellentes rééditions, sous la direction de $H$. Coulet, de romans de femmes du XVIIle siècle, chez Desjonquières.

3 No. 10, 1968, prés. Y. Belaval.

4 Diderot, Oeuvres complètes, éd. Lewinter (Club français du livre, t. X, 1971), p. 32.

5 Cf. Grellet-Dumazeau, Le Salon de Mme Duplessy.

6 Cf. Rouget de Castera, La société toulousaine au XVIllème siècle.

7 On se reportera, évidemment, à l'étude fondamentale de J. Sareil sur Les Tencin (Droz).

8 Sur Mme du Deffand, on consultera, outre les diverses éditions qui ont été données de sa correspondance, l'ouvrage de L. Duisit, Mme du Deffand épistolière (Droz, 1939), et celui de G. Rageot, Mme du Deffand (Albin Michel, 1937).

9 E. et J. de Goncourt, La femme au dix-huitième siècle (rééd. Flammarion, coll. 'Champs,' 1982), pp. 75-77.

10 Voir Correspondance complète de Mme la marquise du Deffand avec ses amis, préface par M. de Lescure (Slatkine reprints, 1971).

11 Cf. Brunel, Les salons, dans Histoire de la littérature française, t. VI.

12 Bohème littéraire et Révolution (trad., Flammarion - CNRS).

13 (Londres, 1774), cité par L. Abensour, La femme et le féminisme avant la Révolution, p. 390.

14 Outre celui que nous venons de citer, Les quatre grades complets de la Francmaçonnerie des dames (Jérusalem, 1772) et L'Adoption ou la Maçonnerie des dames (1775).

15 Cf. Mme du Hausset, Mémoires, cité par L. Abensour, op. cit. p. 279. Voir à ce sujet le dossier DXIX des Archives Nationales.

16 Archives départementales, Orne, cité par L. Abensour.

17 Là encore ce sont les archives départmentales qui nous renseignent et qui devraient être plus systématiquement explorées.

18 L'ouvrage de L. Abensour est particulièrement intéressant sur toute cette question.

19 Cf. Coquerel, Histoire des églises du désert, et les dépêches de M. De SaintFlorentin, intendant du Languedoc.

20 E. Badinter, Émilie, Émilie, l'ambition féminine au XVIIIème siècle (Mercure de France, 1983). Bien entendu, on se reportera à l'édition des Lettres de la marquise du Châtelet publiées par Th. Besterman, 2 vol. (Genève: Institut Voltaire, 
1958), et à l'édition qu'a donnée R. Mauzi de son Discours sur le bonheur, précédée d'une importante préface (Les Belles Lettres, 1961). L'ouvrage de I.O. Wade demeure essentiel, Voltaire and Mme du Châtelet, An Essay on the Intellectual Activity at Cirey (Princeton, 1941). On lira avec intérêt, R. Vaillot, Madame du Châtelet, préface R. Pomeau (Albin Michel, 1978).

21 Lettre du 9 juillet 1744.

22 Lettre à Maupertuis, éd. Besterman, t. I, p. 216.

23 Édition R. Mauzi, p. 21.

24 Publié par I.O. Wade, Voltaire et Mme du Châtelet, appendice, p. 231.

25 Voltaire et Mme du Châtelet, p. 232.

26 Éd. Berterman, t. II, pp. 306-7.

27 Besterman, t. I, p. 16.

28 Besterman, p. 16.

29 B.N. Yp4717.

30 Trad. Duperron et Castera, 2 vol. (Montalant, 1738). Le livre d'Algarotti était dédié à Mme du Châtelet.

31 Mme Thiroux d'Arconville, Mélanges de littérature, de morale et de physique (Amsterdam, 1775).

32 Octavie Durey de Meynières, Mme Belot.

33 Outre Des causes de la corruption du goût (Rigaud, 1714), et Homère défendu contre l'A pologie du R.P. Hardouin (Coignard, 1716), citons sa traduction de l'Iliade et de l'Odyssée, mais aussi celles d'Aristophane, de Cornelius Victor, de Dictys de Crète, d'Eutrope, de Florus, de Marc-Aurèle, de Plaute et de Térence.

34 Anne-Marie Lepage, Mme Fiquet du Bocage, outre les Amazones, tragédie (F. Mérigot, 1749) est l'auteur de nombreux poèmes recueillis dans ses Oeuvres poétiques (Nyon aîné, 1788) du poème La Colombiade, ou la Foi portée au nouveau monde (Desaint et Saillant, 1756), de La mort d'Abel, imité de Gessner, du Paradis terrestre, imité de Milton (Londres, 17xx). Elle a encore traduit Pope.

35 Le Théâtre de Marie-Justine-Benoîte Duronceray, Mme Favart a connu plusieurs éditions (Duchesne, 1763, 8 vol.; Duchesne, 1763-72, 10 vol.). Elle est l'auteur des Amours de Bastien et de Bastienne, parodie du Devin du Village, que Mozart a connu quand il écrit Bastien et Bastienne.

36 Anne-Hyacinthe de Saint-Léger, dame de Colleville. Les deux soeurs ont paru chez Ballard et fils en 1783. Elle est l'auteur d'autres comédies et de romans.

37 Mémoires écrits par elle-même (Londres, 1755), 4 vol.

38 On se reportera à l'édition moderne: Pseudo-mémoires de Madame d'Épinay, Histoire de Mme de Montbrillant, texte intégral, introd. et notes de G. Roth, 3 vol. (Gallimard, 1951).

39 Mlle de Lespinasse, Lettre inédites, préf. Ch. Henry, 1887 (Slatkine, 1971). Nombreuses éditions de la correspondance de Mlle de Lespinasse au XIXème siècle: Correspondance entre Mlle de Lespinasse et le Cte de Guibert (Lévy, 1906).

40 Correspondance complète de Mme la marquise du Deffand avec ses amis, préface de M. de Lescure (Slatkin reprints, 1971). 
41 Oeuvres de Mme d'Épinay, réimpr. sur l'éd. de Genève, 1759 (A. Sauton, 1869): T. I, Lettres à mon fils, t. II, Mes moments heureux. Mémoires et correspondance de Madame d'Épinay, 3 vol. (Brunet, 1818).

42 Oeuvres complètes de Mme la marquise de Lambert (L. Collin, 1808); Avis d'une mère à son fils et à sa fille (Ganeau, 1728); Réflexions nouvelles sur les femmes (Le Breton, 1727); Traité de l'Amitié in Recueil de divers écrits sur l'amour et l'amitié (Bruxelles, 1736).

43 Rééd. Desjonquières.

44 Les malheurs de l'amour; Éléonore d'Yvrée (Guérout, 1687); Inès de Cordoue, nouvelle espagnole (Jouvenel, 1696).

45 Mme Riccoboni, Lettres de Milady Juliette Catesby, roman rééd. avec préface par S. Menant (Desjonquières, 1983).

46 Marie-Anne Roumier, Mme Robert, Voyages de Mylord Céton dans les sept planètes (La Haye, 1765-66). Mme Robert est aussi l'auteur de Nicole de Beauvais ou l'amour vaincu par la reconnaissance (Desaint, 1767), des Ondins, conte moral (Delalain, 1768), de La Paysanne philosophe (Amsterdam, 1761-62), de La Voix de la Nature, aventures de la marquise de... (Amsterdam, 1763).

47 Rééd. Desjonquières (1983), pp. 29 et 60.

48 L. Abensour, p. 313. 